\title{
Organize Sanayi Bölgesi (Uşak) Atıklarından Kurşun (Pb) Biyobirikimi Yapabilen Bakterilerin İzolasyonu ve İdentifikasyonu
}

\section{Ferruh AŞÇI ${ }^{1}$, Gülderen UYSAL AKKUŞ², Safiye Elif KORCAN ${ }^{3}$, Büşra AYDIN ${ }^{1}$, Gamze Kübra ÇETiN ${ }^{1}$, Nazife ALPASLAN ${ }^{1}$}

${ }^{1}$ Afyon Kocatepe Üniversitesi, Fen - Edebiyat Fakültesi, Moleküler Biyoloji ve Genetik Bölümü, Afyonkarahisar.

${ }^{2}$ Afyon Kocatepe Üniversitesi, Fen - Edebiyat Fakültesi, Kimya Bölümü, Afyonkarahisar.

${ }^{3}$ Uşak Üniversitesi, Sağıık Hizmetleri Meslek Yüksekokulu, Uşak.

Sorumlu yazar e-posta: $f$ _asci@aku.edu.tr
guakkus@aku.edu.tr
elif.korcan@usak.edu.tr
cnkaya21@hotmail.com
gamzekubra.ctn@gmail.com
alpaslannazifenaz@gmail.com

ORCID ID: https://orcid.org/0000-0002-9367-6477

Geliş Tarihi: 21.02.2019; ～Kabul Tarihi: 01.10.2019

Anahtar kelimeler Biyobirikim; Kurşun $(\mathrm{Pb})$; Bakteri; 16S rRNA
Öz

Bu çalışmada Uşak ili sınırları içinde bulunan Seramik fabrikası atık örneklerinden dökme plak yöntemi kullanılarak 16 bakteri izolatı elde edildi ve kurşun $(\mathrm{Pb})$ birikimi yapabilen 6 bakterinin identifikasyonu yapıldı. İzolatların $16 \mathrm{~S}$ rRNA sekans analizi yapıldı ve ağır metal birikimi yapabilen bakteriler tespit edildi; SAB2: \%99 Enterobacter cancerogenus; Pantoea agglomerans; Enterobacter cloacae, SÇB9: \%100 Bacillus mycoides; Bacillus cereus; Bacillus weihenstephanensis, SAB4: \%99 Acinetobacter haemolyticus, SAB6: \%100 Pseudomonas aeruginosa, SAB7:\%100 Bacillus subtilis; Bacillus halotolerans; Bacillus mojavensis, SAB9: \%100 Bacillus aryabhattai; Bacillus meqaterium. ICP analiz sonuçlarına göre Bacillus mycoides (\% 57) ve Bacillus aryabhattai'nin (\% 46) kurşun biyosorbsiyonu yapabildiği, ilk kez bu çalışma ile tespit edildi. FTIR analiz sonuçları, amin gruplarının biyosorbsiyonda etkin olduğunu göstermektedir.

\section{Isolation and Identification of Bacteria Capable of Bioaccumulation of Lead (Pb) from Organized Industrial Zone (Usak) Wastes}

\footnotetext{
Abstract

In this study, 16 bacterial isolates were obtained by using pour plate method from ceramic factory

Keywords

Bioaccumulation;

Lead $(\mathrm{Pb})$;

Bacteria;

16S rRNA \begin{abstract}
waste samples located in Uşak province. The identification of 6 bacteria capable of lead $(\mathrm{Pb})$ accumulation was performed. 16S rRNA sequence analysis of the isolates were performed and bacteria capable of heavy metal accumulation were detected; SAB2: \%99 Enterobacter cancerogenus; Pantoea agglomerans; Enterobacter cloacae, SÇB9: \%100 Bacillus mycoides; Bacillus cereus; Bacillus weihenstephanensis, SAB4: \%99 Acinetobacter haemolyticus, SAB6: \%100 Pseudomonas aeruginosa, SAB7:\%100 Bacillus subtilis; Bacillus halotolerans; Bacillus mojavensis, SAB9: \%100 Bacillus aryabhattai, Bacillus meqaterium. According to ICP analysis results, for the first time, Bacillus mycoides (57\%) and Bacillus aryabhattai (46\%) were found to be capable of lead biosorption. FTIR analysis results show that amine groups are effective in biosorption.
\end{abstract}




\section{Giriş}

Ağır metaller içerisinde yer alan kurşun, özellikle endüstriyel atıklarda yaygın bir şekilde bulunmaktadır. Kurşun, yüksek toksisiteye sahiptir. Özellikle kurşun içeren endüstüriyel atıkların kontrolsüz doğaya salınımı kurşunun ekosistemde birikimine ve yayılımına neden olarak ekosistemde var olan canlılar için büyük risk oluşturmaktadır. Ayrıca organizmada birçok enzim ile etkileşime girer. Eğer kanda belirli bir düzeye ulaşırsa karaciğer, böbrek özellikle de kemik ve dişlerde birikmekte ve kalsiyumun yerine apatitin yapısına girmektedir. Kurşunun kanda yarılanma ömrü 20-40, kemiklerde ise 10 yıldır. Yarı ömrünün uzun olması nedeniyle kurşun kirliliğinin yoğun olduğu yerlerde yaşayan insanlarda yaş ilerlemesine bağı olarak vücuttaki kurşun miktarı da artmaktadır (Özbek vd. 1993). Kurşun $(\mathrm{Pb})$ kontamine toprakta yetişen bitkilerde çeşitli klorozlar kök ve kök üstü organlarda büyüme zararları görülür. Topraktaki kurşun miktarı 1-200 $\mu \mathrm{g} / \mathrm{g}$ arasında olup nehir, yağmur ve kar sularıyla yeryüzü sularına ya da topraktan sızmak suretiyle yer altı sularına karışabilmektedir. Ayrıca bitkiler tarafından alınmak suretiyle gıdalarda da birikebildiği yapılan çalışmalarla gösterilmiştir (Kloke vd. 1984; Blume and Hellriegel 1981).

Son yıllarda $\mathrm{Pb}$ 'nin yol açtığı toprak kirliliği tüm dünyanın ortak problemi haline gelmiştir (Başkaya ve Kocaer 2003; Heil vd. 1999). Kirlenmiş toprakların temizlenmesi amacıyla kimyasal arıtım, toprak yıkama, elektrokinetik arıtım, biyoremediasyon ve fitoremediasyon gibi bazı iyileştirme teknikleri geliştirilmiştir (Moutsatsou vd. 2006). Bu nedenle $\mathrm{Pb}^{\prime}$ un biyoremediasyonu için büyük çaba harcanmaktadır.

Biyoremediasyon, kirleticileri ortadan kaldırmak için mikroorganizma metabolizmasının kullanılmasıdır. Bakterileri de içeren doğal mikrobik suşlar, gelişimleri ve metabolizmaları üzerinde herhangi bir etki yaratmadan çok yüksek düzeylerde korunmaya devam etmek için çeşitli koruyucu mekanizmalar kullanırlar. Yüksek konsantrasyonlarda kurşuna direnmek için effluks mekanizmaları, hücre dışı sekretasyon, biyosorpsiyon, çökeltme, hücre morfolojisindeki değişiklik, gelişmiş siderofor üretimi ve hücre içi biyoakümülasyonu gibi çeşitli stratejiler geliştirmişlerdir (Nies 1999; Naik and Dubey 2013). Bu durum, benzersiz özelliklere sahip olan bakteri suşlarının kurşunla kirlenmiş çevresel alanların biyoremediasyonunda ideal bir teknik olduğunu kanıtlamaktadır.

Biyobirikim, bir ortamda çözünmüş bileşenlerin canlı hücrelerin içinde kontrollü bir şekilde hücre zarından geçmek suretiyle birikmesidir (Açıkel 2003). Mikroorganizmalarda metal taşıma mekanizması hücre zarı üzerindeki elektrokimyasal gradientlerle ilişkilidir (Garnham vd. 1992). Mikrobiyal biyobirikimde metaller ilk aşamada iyon değişimi veya fiziksel adsorpsiyon ile hızlı bir şekilde hücre yüzeyinde toplanmaktadır. Daha sonra hücre zarından metabolizmaya bağlı olarak hücre içerisine alınmaktadır. Membranda bulunan tiyollerin, metallerin aktif taşınmasında görev aldığı bildirilmiştir. Hücre içinde bulunan metallotiyonein proteinleri, metalleri şelatlanarak detoksifiye eder (Srinath vd. 2002; Mejare and Bulow 2001).

Streptomyces, Bacillus ve Pseudomonas gibi mikroorganizmalar kontamine sulardaki metalleri akümüle edebilirler. Günümüzde bu amaçla Acinetobacter, Corynebacterium, Flavobacterium, Micrococcus Achromobacter, Alcaligenes, Arthrobacter, Bacillus, Mycobacterium, Norcardia, Pseudomonas, Vibrio, Rhodococcus ve Sphingomonas aktif olarak kullanılan türlerdir (Roychowdhury vd. 2019). Toksik metallere dirençli mikroorganizmalar kontamine sularda biyotoplayıcı olarak kullanılabilir. Bu nedenle bu tür izolatların belirlenmesi hem ekolojik hem de ekonomik açıdan büyük önem taşır. Bu çalışmada Organize Sanayi Bölgesi (Uşak) seramik çamuru (SÇ) ve seramik atıklarından ( $\mathrm{SA}$ ) $\mathrm{Pb}$ biyobirikimi yapabilen metal dirençli bakterilerin izolasyonu ve identifikasyonun yapılması amaçlanmıştır.

\section{Materyal ve Yöntem}

\subsection{Organize Sanayi Bölgesi (Uşak) Atık Örneklerinden Bakteri İzolasyonu ve Kurşun Tuzlarına Dirençli İzolatların Belirlenmesi}


Seramik fabrikası atık örneklerinden Dökme Plak Yöntemi kullanılarak mikroorganizma izolasyonu yapıldı. Besiyeri olarak Nutrient Agar (NA) ve Plate Count Agar (PCA) kullanıldı. İnokülasyon sonrası $37^{\circ} \mathrm{C} 48$ saat, inkübasyona bırakılarak plaklarda oluşan kolonilerin saf kültürleri metal direnci deneylerinde kullanıldı. İzolatlar $\mathrm{Pb}\left(\mathrm{NO}_{3}\right)_{2}$ ' In 0.012510 ppm konsantrasyon aralığında agar ortama eklenerek (nişasta $3 \mathrm{~g} \mathrm{~L}^{-1}$, malt ekstrat $15 \mathrm{~g} \mathrm{~L}^{-1}$ ve agar $\left.20 \mathrm{~g} \mathrm{~L}^{-1}\right) 37 \pm 2^{\circ} \mathrm{C}^{\prime}$ de, 2 gün inkübasyonun ardından ağır metal içermeyen ve farklı konsantrasyonlarda ağır metal tuzları içeren plaklardaki bakteriyel gelişim değerlendirilmiştir.

\section{2 İzolatların Moleküler İdentifikasyonu}

\section{DNA izolasyonu}

DNA izolasyonu (High Pure PCR Template Preparation) kiti ile gerçekleştirildi. 1,5 ml'lik ependorf tüplere $200 \mu \mathrm{l}$ su ya da fosfat tamponu (PBS) içine koloniden pipet ucu ile örnek alındı. 500 rpm de 5 dakika santrifüj edildi ve $200 \mu \mathrm{dH} 2 \mathrm{O}$ eklenerek örnekler çalışma için hazırlandı. Üzerine 5 $\mu$ l litikaz eklenerek iyice karıştırıldı ve $37^{\circ} \mathrm{C}^{\prime}$ de $15 \mathrm{dk}$ inkübasyona bırakıldı. İnkübasyon sonrası $200 \mu \mathrm{l}$ bağlama tamponu ve $40 \mu$ l proteinaz $\mathrm{K}$ eklenerek 10 $\mathrm{dk} 72^{\circ} \mathrm{C}^{\prime}$ de inkübasyona bırakıldı. DNA'yı çöktürmek için karışıma $100 \mu$ lisopropanol ilave edildi. Karışım tüplere aktarıldı ve 8000x g'de $1 \mathrm{dk}$ santrifüj edildi. Bu aşamanın ardından filtreli tüpler değiştirilerek tüpe $500 \mu \mathrm{l}$ inhibitör uzaklaştırma tamponu (IRB) eklenerek 8000 x g'de $1 \mathrm{dk}$ santrifüj edildi. Filtreli tüp tekrar yeni bir tüpe alınarak $500 \mu$ yıkama tamponu (WB) eklendi ve 8000x g'de 1 dk santrifüj edildi. Bu aşama $500 \mu \mathrm{l}$ WB ilavesi ile tekrarlandı. Ardından tüpteki sıvı dökülüp $13000 x$ g' de 10 saniye santrifüj yapılarak filtreli tüpler $72 \mathrm{oC}^{\prime}$ de bekleyen elüsyon tamponu (EB) dan $200 \mu$ l eklenerek 1,5 ml'lik ependorflara alındı. 8000x g' de $1 \mathrm{dk}$ santrifüj yapıldı.

\section{DNA Saflık Kontrolü}

Elde edilen genomik DNA'ların bütünlükleri agaroz jel elektroforezinde, saflık kontrolleri ve miktar tayinleri spektrofotometrik olarak Thermo
Scientific-Nanodrop 2000c cihazı ile yapılmıştır. Bu amaçla $1,5 \mu \mathrm{l}$ DNA örneğinin $A_{260} / A_{280}$ oranı hesaplandı.

\section{PZR (Polimeraz Zincir Reaksiyonu)}

16S rRNA gen fragmentinin amplifikasyonu evrensel primerler kullanılarak Taq DNA Polimeraz Kiti (HelixAmpTM) ile gerçekleştirilmiştir. Amplifikasyon tüpüne eklenecek bileşenler ve reaksiyon basamakları kit içeriğine göre düzenlenmiş olup (Çizelge 1)'de belirtildiği gibidir.

Çizelge 1. PZR bileşenleri ve miktarları

\begin{tabular}{ll}
\hline Bileşen & Miktarı \\
\hline Kalıp DNA & $5 \mu \mathrm{l}$ \\
10X TaqBuffer & $5 \mu \mathrm{l}$ \\
D9NTP & $1 \mu \mathrm{l}$ \\
Primer - F & $1 \mu \mathrm{l}$ \\
Primer - R & $1 \mu \mathrm{l}$ \\
5X TuneUpBuffer & $10 \mu \mathrm{l}$ \\
TaqPolimeraz & 1,25 unit \\
Su & $50 \mu l$ lye tamamlandı \\
\hline
\end{tabular}

Tüm moleküler analizler boyunca Internal Transcribed Spacer (ITS) geninin çoğaltılması için kullanılan koşullar (Çizelge 2)'de gösterilmiştir.

Çizelge 2. 16S rRNAgeninin PZR koşulları

\begin{tabular}{|c|c|c|c|}
\hline İşlem & Döngü & Süre & Sıcaklık \\
\hline İlk denatürasyon & 1 & $2 \mathrm{dk}$ & $95 \div \mathrm{C}$ \\
\hline Denatürasyon & & $20 s n$ & $95 \div \mathrm{C}$ \\
\hline Annealing & 35 & $40 s n$ & $55 \stackrel{\circ}{C}$ \\
\hline Elongasyon & & $1 \mathrm{dk} 30 \mathrm{sn}$ & $72^{\circ} \mathrm{C}$ \\
\hline Son Uzama & 1 & $5 \mathrm{dk}$ & $72^{\circ} \mathrm{C}$ \\
\hline Soğutma & & Sınırsız & $4 \circ \mathrm{C}$ \\
\hline
\end{tabular}

\section{Agaroz jel elektroforezi}

Agaroz jel elektroforezi, 2,5 $\mu \mathrm{l} \mathrm{GelRed'li} \mathrm{\% 1} \mathrm{agaroz}$ (Sigma) içeren mini jelde gerçekleştirildi. Jel hazırlanmasında ve elektroforezde 1xTBE tamponu kullanıldı. $2 \mu \mathrm{l}$ DNA solüsyonu, $1 \mu \mathrm{l}$ yükleme boya solüsyonu ve $3 \mu$ su ile karışımı hazırlanarak 90 V'da 60 dk süreyle yürütüldü. Elektroforezde, DNA markerı olarak fermentas DNA Ladder kullanıldı.

\section{İndüktif Eşleşmiş Plazma Optik Emisyon Spektroskopisi (ICP-OES) ile Pb Biyosorpsiyonunun Belirlenmesi}

Biyosorpsiyon çalışması için kullanılacak bütün cam malzemeler \%5' lik nitrik asitle yaklaşık 5 saat 
bekletilerek ardından distile suda ile bir gece boyunca bekletildi. Kullanmadan önce etüvde kurutuldu (Moore 2007). İzolatlar biyosorpsiyon çalışmasında kullanılmadan önce $100 \mathrm{ml} \mathrm{NB}$ besiyeri içeren 250 ml'lik erlenlere ekimleri yapıldı ve gelişmeleri için çalkalamalı inkübatörde (125rpm) $27^{\circ} \mathrm{C}^{\prime} \mathrm{de}$ inkübe edildi. İnkübasyondan sonra biyomas steril filtre kağıtlarından süzülerek. Süzülen biyomas steril distile suyla besiyeri kalıntısı olmaması için yıkandı. Biyomaslar 2,5 g tartılarak erlenlere konularak. Biyomas ve 100 ppm konsantrasyonda $\mathrm{Pb}$ bulunan erlenler $25{ }^{\circ} \mathrm{C}^{\prime}$ de 125 rpm' de çalkalamalı etüvde biyosorpsiyon için bırakıldı. Belirli zaman aralıklarında analiz için ağır metal solüsyonları alındı. Metal solüsyonunun biyomastan ayrılması için 8000 rpm' de 30 dakika santrifüj edildi. Ayrılan ağır metal kısmı (sıvı kısım) falkonlara konularak ICP-OES cihazında analiz edildi.

Ağır metal konsantrasyon verilerine göre (ICP analizi sonuçları) yüzde (\%) olarak biyosorpsiyon verilen formüle göre hesaplandı;

$R=(P 0-P e / P o) \times 100$

$R=$ Yüzde (\%) biyosorpsiyon

PO = Metal iyonları başlangıç konsantrasyonu $(\mathrm{mg} / \mathrm{lt})$

$\mathrm{Pe}=$ Metal iyonlarının kalan konsantrasyonu $(\mathrm{mg} / \mathrm{lt})$ (Amini et al. 2008)

Fourier Transform Infrared spektroskopisi (FTIR) Analizi

Biyokütle oda sıcaklığında vakumla buharlaştırıcı ile kurutulduktan sonra. $100 \mathrm{mg} \mathrm{KBr}$ içinde yaklaşık 1 mg biyokütle kapsüllendi ve Fourier Transform Infrared spektroskopisi (Perkin Elmer Spektrum 100) ile analiz edildi.

\section{Bulgular}

Seramik fabrikası; Seramik Çamuru (SÇ) ve Seramik Atıkları (SA) örneklerindeki toplam mezofilik bakteri sayısı (Çizelge 3)' de verilmiştir. SA ve SÇ'den yapılan ekimlerde en fazla mikroorganizma NA besiyerinde elde edilmiştir. Besiyerindeki koloni morfolojisine bakılarak birbirine benzemeyen koloniler seçilmiş ve bundan sonraki denemelerde kullanılmıştır. 18 izolatın 0.0125 ila $10 \mathrm{mM}$ konsantrasyon aralığında kurşun içeren ortamda üreyebildiği saptanmıştır (Çizelge 4). Bazı izolatların

(SÇB3,5 ve SAB2) yüksek $\mathrm{Pb}$ konsantrasyonunda üremesi ancak düşük $\mathrm{Pb}$ konsantrasyonlarında ürememiş olması ekim hatalarından kaynaklandığı düşünülmektedir.

Çizelge 3. Seramik fabrikası; Seramik Çamuru (SÇ) ve Seramik Atıkları (SA) örneklerinin $1 \mathrm{ml}$ 'sinde bulunan mikroorganizma sayısı

\begin{tabular}{ccc}
\hline Besiyeri & \multicolumn{2}{c}{ CFU/ml } \\
\cline { 2 - 3 } & $\mathrm{SC}$ & $\mathrm{SA}$ \\
PCA & $8.0 \times 10^{3}$ & $1.6 \times 10^{4}$ \\
NA & $8.0 \times 10^{4}$ & $2.9 \times 10^{7}$ \\
\hline
\end{tabular}

Çizelge 4. 0.0125 ile $10 \mathrm{mM}$ konsantrasyon aralı̆̆ında kurşun içeren ortamda üreyebilen izolatlar.

\begin{tabular}{|c|c|c|c|c|c|c|c|c|c|}
\hline & $\begin{array}{l}\overline{0} \\
\stackrel{0}{t} \\
\stackrel{0}{0} \\
\underline{y}\end{array}$ & $\frac{\widehat{O}}{\stackrel{\theta}{0}}$ & $\frac{\widehat{n}}{\frac{0}{0}}$ & 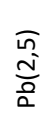 & $\frac{\widehat{D}}{\frac{0}{0}}$ & $\frac{\widehat{n}}{\frac{\tilde{d}}{0}}$ & $\frac{\bar{\theta}}{\frac{0}{\alpha}}$ & $\begin{array}{l}\widehat{\tilde{n}} \\
\frac{0}{0} \\
\frac{0}{2}\end{array}$ & $\begin{array}{l}\text { 今n } \\
\text { - } \\
0 \\
0 \\
0 \\
\frac{0}{0}\end{array}$ \\
\hline SAB1 & + & + & + & + & + & + & + & + & + \\
\hline SAB2 & + & + & + & + & + & - & + & + & + \\
\hline SAB3 & + & + & + & + & + & + & + & + & + \\
\hline SAB4 & + & + & + & + & + & + & + & + & + \\
\hline SAB5 & + & + & + & + & + & + & + & + & + \\
\hline SAB6 & + & + & + & + & + & + & + & + & + \\
\hline SAB7 & + & + & + & + & + & + & + & + & + \\
\hline SAB8 & + & + & + & + & + & + & + & + & + \\
\hline SAB9 & + & + & + & + & + & - & + & + & + \\
\hline SÇB1 & + & + & + & + & + & + & + & + & + \\
\hline SÇB2 & + & + & + & + & + & + & + & + & + \\
\hline SÇB3 & + & - & + & + & - & + & + & - & - \\
\hline SÇB4 & + & + & + & + & + & + & - & + & + \\
\hline SÇB5 & - & - & + & - & - & - & - & - & - \\
\hline SÇB6 & + & + & + & + & + & + & + & + & + \\
\hline SÇB7 & + & + & + & + & + & + & + & + & + \\
\hline SÇB8 & + & + & + & + & + & + & + & + & + \\
\hline SÇB9 & + & + & + & + & + & + & + & + & + \\
\hline
\end{tabular}




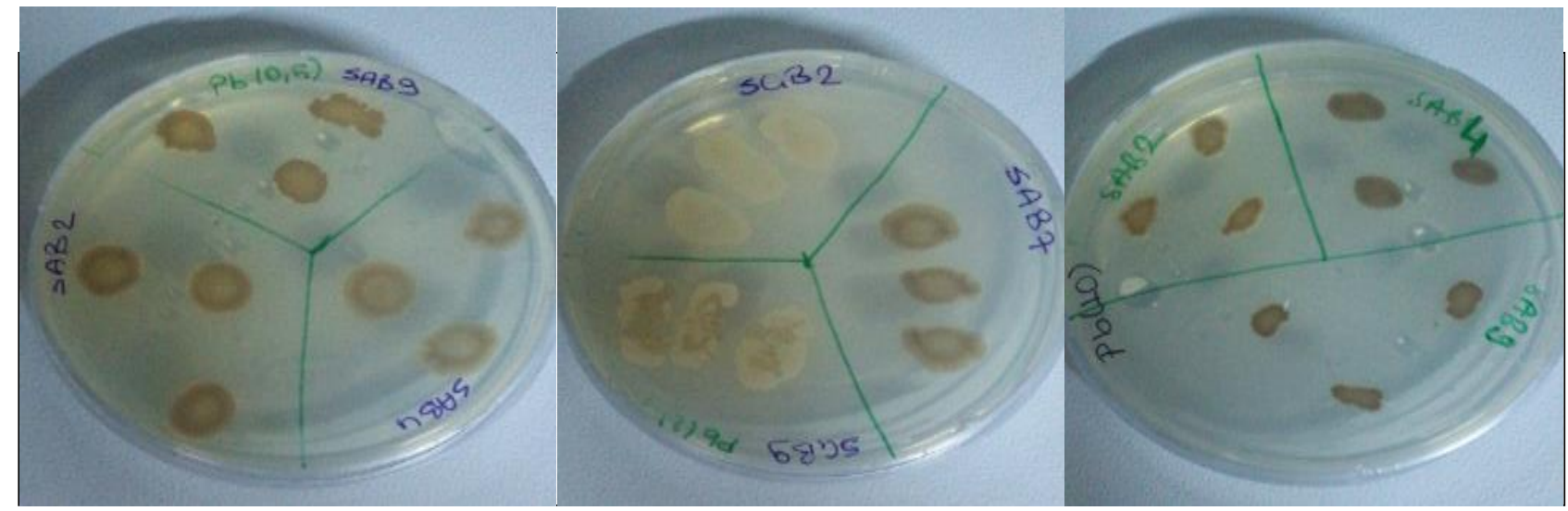

Şekil 1. Biyosorbant izolatların makroskobik morfolojileri

$\mathrm{Pb}$ içeren ortamda koloni renginin kahverengiye dönmesi kurşunun biyosorbsiyonunun yapıldığını göstermektedir. Bu çalış̧mada 6 izolatın (SAB2, SAB4, SAB6, SAB7, SAB9 ve SÇB9) biyosorbsiyon yapabildiği kültür ortamında koloni renk değişimi ile saptanmıştır (Şekil 1). 16S rRNA dizi analizi sonucunda; SAB2: \%99 Enterobacter cancerogenus; Pantoea agglomerans;
Enterobacter cloacae, SÇB9: \%100 Bacillus mycoides; Bacillus cereus; Bacillus weihenstephanensis, SAB4: \%99 Acinetobacter haemolyticus, SAB6: \%100 Pseudomonas aeruginosa, SAB7:\%100 Bacillus subtilis; Bacillus halotolerans; Bacillus mojavensis, SAB9: \%100 Bacillus aryabhattai; Bacillus megaterium olarak identifiye edilmiştir (Çizelge 5).

Çizelge 5. Blast (BLAST) analizi sonucunda izolatın türü 
SAB2: \%99 Enterobacter cancerogenus;

\section{Pantoea agglomerans;}

Enterobacter cloacae
TCGGGTGACGAGTGGCGGACGGGTGAGTAATGTCTGGGAAACTGCCTGATGGAGGGGGAT AACTACTGGAAACGGTAGCTAATACCGCATAACGTCGCAAGACCAAAGAGGGGGACCTTCG GGCCTCTTGCCATCAGATGTGCCCAGATGGGATTAGCTAGTAGGTGGGGTAACGGCTCACCT AGGCGACGATCCCTAGCTGGTCTGAGAGGATGACCAGCCACACTGGAACTGAGACACGGTC CAGACTCCTACGGGAGGCAGCAGTGGGGAATATTGCACAATGGGCGCAAGCCTGATGCAGC CATGCCGCGTGTATGAAGAAGGCCTTCGGGTTGTAAAGTACTTTCAGCGGGGAGGAAGGTG TTGTGGTTAATAACCRCAGCAATTGACGTTACCCGCAGAAGAAGCACCGGCTAACTCCGTGCC AGCAGCCGCGGTAATACGGAGGGTGCAAGCGTTAATCGGAATTACTGGGCGTAAAGCGCAC GCAGGCGGTCTGTCAAGTCGGATGTGAAATCCCCGGGCTCAACCTGGGAACTGCATTCGAAA CTGGCAGGCTAGAGTCTTGTAGAGGGGGGTAGAATTCCAGGTGTAGCGGTGAAATGCGTAG AGATCTGGAGGAATACCGGTGGCGAAGGCGGCCCCCTGGACAAAGACTGACGCTCAGGTGC GAAAGCGTGGGGAGCAAACAGGATTAGATACCCTGGTAGTCCACGCCGTAAACGATGTCGA CTTGGAGGTTGTGCCCTTGAGGCGTGGCTTCCGGAGCTAACGCGTTAAGTCGACCGCCTGGG GAGTACGGCCGCAAGGTTAAAACTCAAATGAATTGACGGGGGCCCGCACAAGCGGTGGAGC ATGTGGTTTAATTCGATGCAACGCGAAGAACCTTACCTACTCTTGACATCCAGAGAACTTASC AGAGATGSWTTGGTGCCTTCGGGAACTCTGAGACAGGTGCTGCATGGCTGTCGTCAGCTCGT GTTGTGAAATGTTGGGTTAAGTCCCGCAACGAGCGCAACCCTTATCCTTTGTTGCCAGCGGTY MGGCCGGGAACTCAAAGGAGACTGCCAGTGATAAACTGGAGGAAGGTGGGGATGACGTCA AGTCATCATGGCCCTTACGAGTAGGGCTACACACGTGCTACAATGGCGCATACAAAGAGAAG CGACCTCGCGAGAGCAAGCGGACCTCATAAAGTGCGTCGTAGTCCGGATTGGAGTCTGCAAC TCGACTCCATGAAGTCGGAATCGCTAGTAATCGTAGATCAGAATGCTACGGTGAATACGTTCC CGGGCCTTGTACACACCGCCCGTCACACCATGGGAGTGGGTTGCAAAAGAAGTAGGTA

GAAGTTAGCGGCGGACGGGTGAGTAACACGTGGGTAACCTACCCATAAGACTGGGATAACT CCGGGAAACCGGGGCTAATACCGGATAATATTTTGAACTGCATAGTTCGAAATTGAAAGGCG GCTTCGGCTGTCACTTATGGATGGACCCGCGTCGCATTAGCTAGTTGGTGAGGTAACGGCTC ACCAAGGCGACGATGCGTAGCCGACCTGAGAGGGTGATCGGCCACACTGGGACTGAGACAC GGCCCAGACTCCTACGGGAGGCAGCAGTAGGGAATCTTCCGCAATGGACGAAAGTCTGACG GAGCAACGCCGCGTGAGTGATGAAGGCTTTCGGGTCGTAAAACTCTGTTGTTAGGGAAGAA CAAGTGCTAGTTGAATAAGCTGGCACCTTGACGGTACCTAACCAGAAAGCCACGGCTAACTA CGTGCCAGCAGCCGCGGTAATACGTAGGTGGCAAGCGTTATCCGGAATTATTGGGCGTAAA GCGCGCGCAGGTGGTTTCTTAAGTCTGATGTGAAAGCCCACGGCTCAACCGTGGAGGGTCAT TGGAAACTGGGAGACTTGAGTGCAGAAGAGGAAAGTGGAATTCCATGTGTAGCGGTGAAAT GCGTAGAGATATGGAGGAACACCAGTGGCGAAGGCGACTTTCTGGTCTGTAACTGACACTG AGGCGCGAAAGCGTGGGGAGCAAACAGGATTAGATACCCTGGTAGTCCACGCCGTAAACGA TGAGTGCTAAGTGTTAGAGGGTTTCCGCCCTTTAGTGCTGAAGTTAACGCATTAAGCACTCCG CCTGGGGAGTACGGCCGCAAGGCTGAAACTCAAAGGAATTGACGGGGGCCCGCACAAGCG GTGGAGCATGTGGTTTAATTCGAAGCAACGCGAAGAACCTTACCAGGTCTTGACATCCTCTG AAAACTCTAGAGATAGAGCTTCTCCTTCGGGAGCAGAGTGACAGGTGGTGCATGGTTGTCGT CAGCTCGTGTCGTGAGATGTTGGGTTAAGTCCCGCAACGAGCGCAACCCTTGATCTTAGTTGC CATCATTAAGTTGGGCACTCTAAGGTGACTGCCGGTGACAAACCGGAGGAAGGTGGGGATG ACGTCAAATCATCATGCCCCTTATGACCTGGGCTACACACGTGCTACAATGGACGGTACAAAG AGCTGCAAGACCGCGAGGTGGAGCTAATCTCATAAAACCGTTCTCAGTTCGGATTGTAGGCT GCAACTCGCCTACATGAAGCTGGAATCGCTAGTAATCGCGGATCAGCATGCCGCGGTGAATA CGTTCCCGGGCCTTGTACACACCGCC

AGTGTAGCTTGCTACATTACCTAGCGGCGGACGGGTGAGTAATGCTTAGGAATCTGCCTATT AGTGGGGGACAACATTCCGAAAGGAATGCTAATACCGCATACGTCCTACGGGAGAAAGCAG GGGATCTTCGGACCTTGCGCTAATAGATGAGCCTAAGTCGGATTAGCTAGTTGGTGGGGTAA AGGCCTACCAAGGCGACGATCTGTAGCGGGTCTGAGAGGATGATCCGCCACACTGGGACTG AGACACGGCCCAGACTCCTACGGGAGGCAGCAGTGGGGAATATTGGACAATGGGSGGAASC CTGATCCAGCCATGCCGCGTGTGTGAAGAAGGCCTTATGGTTGTAAAGCACTTTAAGCGAGG AGGAGGCTACTCTAGTTAATACCTAGAGATAGTGGACGTTACTCGCAGAATAAGCACCGGCT AACTCTGTGCCAGCAGCCGCGGTAATACAGAGGGTGCGAGCGTTAATCGGATTTACTGGGCG TAAAGCGTGCGTAGGCGGCTGATTAAGTCGGATGTGAAATCCCTGAGCTTAACTTAGGAATT GCATTCGATACTGGTCAGCTAGAGTATGGGAGAGGATGGTAGAATT 
SAB6: $\% 100$ Pseudomonas aeruginosa

SAB7: \%100 Bacillus subtilis;

Bacillus halotolerans;

Bacillus mojavensis
AGCGGATGAAGGGAGCTTGCTCCTGGATTCAGCGGCGGACGGGTGAGTAATGCCTAGGAAT CTGCCTGGTAGTGGGGGATAACGTCCGGAAACGGGCGCTAATACCGCATACGTCCTGAGGG AGAAAGTGGGGGATCTTCGGACCTCACGCTATCAGATGAGCCTAGGTCGGATTAGCTAGTTG GTGGGGTAAAGGCCTACCAAGGCGACGATCCGTAACTGGTCTGAGAGGATGATCAGTCACA CTGGAACTGAGACACGGTCCAGACTCCTACGGGAGGCAGCAGTGGGGAATATTGGACAATG GGCGAAAGCCTGATCCAGCCATGCCGCGTGTGTGAAGAAGGTCTTCGGATTGTAAAGCACTT TAAGTTGGGAGGAAGGGCAGTAAGTTAATACCTTGCTGTTTTGACGTTACCAACAGAATAAG CACCGGCTAACTTCGTGCCAGCAGCCGCGGTAATACGAAGGGTGCAAGCGTTAATCGGAATT ACTGGGCGTAAAGCGCGCGTAGGTGGTTCAGCAAGTTGGATGTGAAATCCCCGGGCTCAAC CTGGGAACTGCATCCAAAACTACTGAGCTAGAGTACGGTAGAGGGTGGTGGAATTTCCTGTG TAGCGGTGAAATGCGTAGATATAGGAAGGAACACCAGTGGCGAAGGCGACCACCTGGACTG ATACTGACACTGAGGTGCGAAAGCGTGGGGAGCAAACAGGATTAGATACCCTGGTAGTCCA CGCCGTAAACGATGTCGACTAGCCGTTGGGATCCTTGAGATCTTAGTGGCGCAGCTAACGCG ATAAGTCGACCGCCTGGGGAGTACGGCCGCAAGGTTAAAACTCAAATGAATTGACGGGGGC CCGCACAAGCGGTGGAGCATGTGGTTTAATTCGAAGCAACGCGAAGAACCTTACCTGGCCTT GACATGCTGAGAACTTTCCAGAGATGGATTGGTGCCTTCGGGAACTCAGACACAGGTGCTGC ATGGCTGTCGTCAGCTCGTGTCGTGAGATGTTGGGTTAAGTCCCGTAACGAGCGCAACCCTT GTCCTTAGTTACCAGCACCTCGGGTGGGCACTCTAAGGAGACTGCCGGTGACAAACCGGAGG AAGGTGGGGATGACGTCAAGTCATCATGGCCCTTACGGCCAGGGCTACACACGTGCTACAAT GGTCGGTACAAAGGGTTGCCAAGCCGCGAGGTGGAGCTAATCCCATAAAACCGATCGTAGTC CGGATCGCAGTCTGCAACTCGACTGCGTGAAGTCGGAATCGCTAGTAATCGTGAATCAGAAT GTCACGGTGAATACGTTCCCGGGCCTTGTACACACCGCCCGTCACACCATGGGAGTGGGTTG CTCCAGAAGTAGCTAGTCTAACCGC

TTGCTCCCTGATGTTAGCGGCGGACGGGTGAGTAACACGTGGGTAACCTGCCTGTAAGACTG GGATAACTCCGGGAAACCGGGGCTAATACCGGATGCTTGTTTGAACCGCATGGTTCAAACAT AAAAGGTGGCTTCGGCTACCACTTACAGATGGACCCGCGGCGCATTAGCTAGTTGGTGAGGT AACGGCTCACCAAGGCAACGATGCGTAGCCGACCTGAGAGGGTGATCGGCCACACTGGGAC TGAGACACGGCCCAGACTCCTACGGGAGGCAGCAGTAGGGAATCTTCCGCAATGGACGAAA GTCTGACGGAGCAACGCCGCGTGAGTGATGAAGGTTTTCGGATCGTAAAGCTCTGTTGTTAG GGAAGAACAAGTACCGTTCGAATAGGGCGGTACCTTGACGGTACCTAACCAGAAAGCCACG GCTAACTACGTGCCAGCAGCCGCGGTAATACGTAGGTGGCAAGCGTTGTCCGGAATTATTGG GCGTAAAGGGCTCGCAGGCGGTTCCTTAAGTCTGATGTGAAAGCCCCCGGCTCAACCGGGG AGGGTCATTGGAAACTGGGGAACTTGAGTGCAGAAGAGGAGAGTGGAATTCCACGTGTAGC GGTGAAATGCGTAGAGATGTGGAGGAACACCAGTGGCGAAGGCGACTCTCTGGTCTGTAAC TGACGCTGAGGAGCGAAAGCGTGGGGAGCGAACAGGATTAGATACCCTGGTAGTCCACGCC GTAAACGATGAGTGCTAAGTGTTAGGGGGTTTCCGCCCCTTAGTGCTGCAGCTAACGCATTA AGCACTCCGCCTGGGGAGTACGGTCGCAAGACTGAAACTCAAAGGAATTGACGGGGGCCCG CACAAGCGGTGGAGCATGTGGTTTAATTCGAAGCAACGCGAAGAACCTTACCAGGTCTTGAC ATCCTCTGACAATCCTAGAGATAGGACGTCCCCTTCGGGGGCAGAGTGACAGGTGGTGCATG GTTGTCGTCAGCTCGTGTCGTGAGATGTTGGGTTAAGTCCCGCAACGAGCGCAACCCTTGATC TTAGTTGCCAGCATTCAGTTGGGCACTCTAAGGTGACTGCCGGTGACAAACCGGAGGAAGGT GGGGATGACGTCAAATCATCATGCCCCTTATGACCTGGGCTACACACGTGCTACAATGGACA GAACAAAGGGCAGCGAAACCGCGAGGTTAAGCCAATCCCACAAATCTGTTCTCAGTTCGGAT CGCAGTCTGCAACTCGACTGCGTGAAGCTGGAATCGCTAGTAATCGCGGATCAGCATGCCGC GGTGAATACGTTCCCGGGCCTTGTACACACCGCCCGT 


\begin{abstract}
TGCAGTCGAGCGACTGATTAGAAGCTTGCTTCTATGACGTTAGCGGCGGACGGGTGAGTAAC ACGTGGGCAACCTGCCTGTAAGACTGGGATAACTTCGGGAAACCGAAGCTAATACCGGATAG GATCTTCTCCTTCATGGGAGATGATTGAAAGATGGTTTCGGCTATCACTTACAGATGGGCCCG CGGTGCATTAGCTAGTTGGTGAGGTAACGGCTCACCAAGGCAACGATGCATAGCCGACCTGA GAGGGTGATCGGCCACACTGGGACTGAGACACGGCCCAGACTCCTACGGGAGGCAGCAGTA GGGAATCTTCCGCAATGGACGAAAGTCTGACGGAGCAACGCCGCGTGAGTGATGAAGGCTT TCGGGTCGTAAAACTCTGTTGTTAGGGAAGAACAAGTACGAGAGTAACTGCTCGTACCTTGA CGGTACCTAACCAGAAAGCCACGGCTAACTACGTGCCAGCAGCCGCGGTAATACGTAGGTGG CAAGCGTTATCCGGAATTATTGGGCGTAAAGCGCGCGCAGGCGGTTTCTTAAGTCTGATGTG AAAGCCCACGGCTCAACCGTGGAGGGTCATTGGAAACTGGGGAACTTGAGTGCAGAAGAGA AAAGCGGAATTCCACGTGTAGCGGTGAAATGCGTAGAGATGTGGAGGAACACCAGTGGCGA AGGCGGCTTTTTGGTCTGTAACTGACGCTGAGGCGCGAAAGCGTGGGGAGCAAACAGGATT AGATACCCTGGTAGTCCACGCCGTAAACGATGAGTGCTAAGTGTTAGAGGGTTTCCGCCCTTT AGTGCTGCAGCTAACGCATTAAGCACTCCGCCTGGGGAGTACGGTCGCAAGACTGAAACTCA AAGGAATTGACGGGGGCCCGCACAAGCGGTGGAGCATGTGGTTTAATTCGAAGCAACGCGA AGAACCTTACCAGGTCTTGACATCCTCTGACAACTCTAGAGATAGAGCGTTCCCCTTCGGGGG ACAGAGTGACAGGTGGTGCATGGTTGTCGTCAGCTCGTGTCGTGAGATGTTGGGTTAAGTCC CGCAACGAGCGCAACCCTTGATCTTAGTTGCCAGCATTTAGTTGGGCACTCTAAGGTGACTGC CGGTGACAAACCGGAGGAAGGTGGGGATGACGTCAAATCATCATGCCCCTTATGACCTGGG CTACACACGTGCTACAATGGATGGTACAAAGGGCTGCAAGACCGCGAGGTCAAGCCAATCCC ATAAAACCATTCTCAGTTCGGATTGTAGGCTGCAACTCGCCTACATGAAGCTGGAATCGCTAG TAATCGCGGATCAGCATGCCGCGGTGAATACGTTCCCGGGCCTTGTACACACCGCCCGTCAC ACCACGAGAGTTTGTAACACCCGAAGTCGGTGG
\end{abstract}

16S rRNA dizi analizi sonucunda SAB2: \%99 Enterobacter cancerogenus; Pantoea agglomerans; Enterobacter cloacae, SÇB9: \%100 Bacillus mycoides; Bacillus cereus; Bacillus weihenstephanensis, SAB4: \%99 Acinetobacter haemolyticus, SAB6: $\% 100$ Pseudomonas aeruginosa, SAB7: \%100 Bacillus subtilis; Bacillus halotolerans; Bacillus mojavensis, SAB9: \%100 Bacillus aryabhattai; Bacillus megaterium olarak identifiye edilmiştir (Çizelge 5).

ICP sonucuna göre, Bacillus mycoides 82. saatte (\%57), Bacillus aryabhattai ise 72 . saatte (\%46) maksimum \% biyosorbsiyon gerçekleştirmiştir (Çizelge 6).

Bu çalışmada FTIR analizi sonuçta, kurşunun biyorpsiyonu ile ilgili olarak fonksiyonel gruplar arasındaki fark belirlenmiştir. Kurşun yüklü mikrobiyal biyokütlenin absorpsiyon spektrumu, kontrol biyokütle ile karşılaştırıldığında, amid gruplarında absorpsiyon bandı (1700-1500 m) değişikliği görülmüştür.
Çizelge 6. 100 ppm Kurşun konsantrasyonlarının ICP sonuçları ve \% biyosorpsiyonları (\%BS)

\begin{tabular}{|c|c|c|c|c|}
\hline & Bacillus & coides & Bacillus & bhattai \\
\hline Zaman (saat) & ICP & $\% \mathrm{BS}$ & ICP & \%BS \\
\hline 2 & 97 & 3 & 93 & 7 \\
\hline 5 & 93 & 7 & 95 & 5 \\
\hline 10 & 82 & 18 & 78 & 22 \\
\hline 15 & 88 & 12 & 73 & 27 \\
\hline 20 & 79 & 21 & 68 & 32 \\
\hline 25 & 72 & 28 & 72 & 28 \\
\hline 30 & 66 & 34 & 62 & 38 \\
\hline 35 & 65 & 35 & 65 & 35 \\
\hline 40 & 65 & 35 & 52 & 48 \\
\hline 45 & 58 & 42 & 53 & 47 \\
\hline 50 & 52 & 48 & 55 & 45 \\
\hline 55 & 47 & 53 & 54 & 46 \\
\hline 60 & 47 & 53 & 58 & 42 \\
\hline 65 & 55 & 45 & 53 & 48 \\
\hline 70 & 42 & 58 & 54 & 46 \\
\hline 75 & 45 & 55 & 54 & 46 \\
\hline 80 & 43 & 57 & - & - \\
\hline 85 & 43 & 57 & - & - \\
\hline
\end{tabular}




\section{Tartışma ve Sonuç}

Biyobirikimi sağlayan metallotiyoneinler, toksik ağır metallerin immobilizasyonunda önemli bir rol oynamakla birlikte ayrıca enzimler tarafından katalize edilen bakteriyel metabolik süreçleri korurlar. (Blindauer vd. 2002; Liu vd. 2003). Bazı siyanobakteriyel, Synechococcus PCC 7942 (SmtA), Anabaena PCC 7120 (SmtA), Oscillatori abrevis (BmtA), Pseudomonas aeruginosa (BmtA) ve Pseudomonas putida (BmtA) izolatlarının sitozolik metal homeostazisini muhafaza etmek için metallotiyoneinlerin kodladığı bildirilmiştir (Blindauer vd. 2002; Turner vd. 1996). Kurşuna maruz kalan Pseudomonas fluorescens' da (Sharma et al., 2006), P. aeruginosa (Naik vd. 2012), Bacillus megaterium'da kurşun direncinden sorumlu metallotiyonein (Roane 1999) gösterilmiştir. Salmonella choleraesuis suşu $4 \mathrm{~A}$ ve Proteuspenneri suşu GM10'da hücre içi biyoakümülasyonunu sağlayan kurşun direncinden sorumlu metal bağlayıcı metallotiyonein smtAB genlerinin (507 bp) varlı̆ı̆ı ortaya çıkardı (Naik vd. 2012). B. megaterium, $P$. aeruginosa suşu $\mathrm{WI}-1, \mathrm{~S}$. Choleraesuis suşu 4A ve $P$. penneri suşu GM10, çok yüksek miktarda kurşunun biyolojik olarak biriktirilebilmesini mümkün kılan, kontamine çevre bölgelerinde bulunan kurşunun biyoremediasyonu için kullanılabilir (Roane 1999).

Biyoremediasyon süreçleri ekonomiktir, çevre dostu ve kontamine çevre bölgelerinden gelen ağır metallerin giderilmesi için fizikokimyasal yöntemlerle kıyaslandığında oldukça verimlidir. Son yıllarda araştırmacılar kurşun biyoremediasyonunda biyoteknolojik ajanlar olarak mikroorganizmaları kullanmaya odaklanmıştı. Bunun nedeni pahalı ve zahmetli kimyasal analiz yöntemlerinin aksine, genlerin füzyonu ile geliştirilen bakteriyel biyo-taşıyıcıların nispeten ucuz, kullanımı kolay, kurşuna özel ve kurşun nanomollerini algılayabilir olmalarıdır.

$\mathrm{Bu}$ çalışmada SAB2 izolatı Enterobacte cancerogenus; Pantoea agglomerans; Enterobacter cloacae, bir izolat (SAB4) Acinetobacter haemolyticus, bir izolat (SAB6)
Pseudomonas aeruginosa, üç izolat ise Basillus sp. [(SÇB9) Bacillus mycoides; Bacillus cereus; Bacillus weihenstephanensis, (SAB7) Bacillus subtilis; Bacillus halotolerans; Bacillus mojavensis, (SAB9) Bacillus aryabhattai; Bacillus megaterium] olarak identifiye edilmiştir (Çizelge 5).

Kurşun kirli atıksulardan Pseudomonas sp., Chryseomonasluteola, Bacillus circulans, Bacillus cereus, Bacillus. subtilis, Bacillus pumilus gibi bakteri kullanarak kurşunun uzaklaştırıması birkaç yazar tarafından bildirilmiştir (Leung vd 2000, Azza vd 2009).Ancak yaptığımız literatür çalışmaları sonucu Bacillus mycoides ve Bacillus aryabhattai nın $\mathrm{Pb}$ biosorbsiyonu yaptığı üzerine hiçbir çalışma bulunmamaktadır. Bu nedenle ICP ve FTIR çalışmaları bu iki Bacillus türünde yapılmıştır.

Bacillus mycoides 82. Saatte (\%57), Bacillus aryabhattai 72. Saatte (\%46) maksimum \%biyosorbsiyon gerçekleştirdiği saptanmıştır. Bizim sonuçlarımıza benzer olarak Arifiyanto vd (2016) Basillus izolatlarının (S1, S11) sırasıyla 53\% ve $51 \% \mathrm{~Pb}$ biyosorbsiyon yapabildiklerini bildirmişlerdir.

Biyosorpsiyon, ayrıca ekzopolisakkaritlerin bakteri hücre yüzeyinde ağır metallerin şelatörü olarak da rol oynadığı bir mekanizmaydı. Bakteriyel hücresinin duvarında, fosforil (PO43-), karboksil (COO-), sülfhidril, tiyol (-SH) ve hidroksil (-OH) gibi negatif yükü içeren grupları bol miktarda bulunur ve bu gruplar ile metal iyonlarını bağlayabilirler etkileşimleri oluşabilir (Murthy vd, 2011)

Son zamanlarda yapılan çalışmalar, karboksil, amin, hidroksil, fosfat ve sülfhidril grupları gibi bazı anyonik hücre yüzey ligandlarının biyosorbent olarak hem canlı hem de ölü bakteri biyokütlesini kullanarak $\mathrm{Pb}$ gibi katyonik metal iyonunun aktif veya pasif biyo-emiliminde kullanılabileceğine dikkat çekmektedir (Murthy vd. 2012).

Bu çalışmada FTIR analizi sonuçta kurşun yüklü mikrobiyal biyokütlenin absorpsiyon spektrumu, kontrol biyokütle ile karşılaştırıldığında, amid gruplarında absorpsiyon bandı değişikliği görülmüştür. Bu nedenle biyosorbsiuonda amin gruplarının etkin olduğu düşünülmektedir. 
Çok yüksek oranda bakteriyel metallotiyonein (BmtA) eksprese eden genetik olarak modifiye edilmiş bakteriler (GMB), kurşunla yüksek oranda kirlenmiş olan çevre bölgelerini biyolojik iyileştirmede kullanılabilir. Bu hiper metal biriktirici bakteriler, metal biyoyararlanımını azaltarak metal homoestazisini korurlar. Hücre yüzey proteinleri ile füzyon, hücre yüzeyinde metallotiyoneinlerin ekspresyonu bakterilerin biyoakümülasyon kapasitesini birkaç kat artırabilir. Bakteriyel suşlar, hücre yüzeyinde veya EPS ve biyosürfaktanlar gibi mikrobiyal ürünler üzerinde yüksek seviyelerde toksik kurşunun biyosorbsiyonu için yüksek seviyelerde metal bağlama ligandları, örneğin karboksil, hidroksil, sülfat, fosfat ve amin eksprese etmek üzere genetik olarak tasarlanabilir. Ağır metal biyoremediasyonunun etkinliği rekombinant mikroorganizmaların uygulanmasıyla üç ila altı kat arttırılabilir (Srivastava and Majumber 2008).

Bir başka yaklaşım, yüksek seviyelerde dirençlere direnç gösterebilen mikrobiyal konsorsiyum kullanılarak endüstriyel atıklardan kurşun çıkarmaya yönelik paketlenmiş yataklı reaktörlerin tasarlanması ve geliştirilmesi olabilir.

$\mathrm{Bu}$ çalışmada Bacillus mycoides ve Bacillus aryabhattai ' $\mathrm{n}$ n Pb biosorbssiyonu yapabildiği ICP ile ilk defa gösterilmiştir. Sonuç olarak kurşun akümüle ettiği saptanan bu izolatların bakteriyel metallotiyonein ekspresyon koşullarının optimizasyonu ile ilgili çalışmaların yapılması hem endüstriyel atıklardan kurşunun yeniden kazanımı için hem de biyoremediasyon uygulamalarında kullanımında büyük faydalar sağlayabilir.

\section{Teşekkür}

Bu çalışma Afyon Kocatepe Üniversitesi Bilimsel Araştırma Projeleri kapsamında yürütülen 17.Kariyer. 98 projesi ile desteklenmiştir.

\section{KAYNAKÇA}

Açıkel, U., 2003. Melas katılan ve ağır metal kirliliği içeren atıksularda çeşitli türdeki maya hücrelerinin büyüme kinetiğinin ve hücrelerdeki metal biyobirikiminin irdelenmesi. Doktora Tezi, Kimya Mühendisliği Anabilim Dalı, Hacettepe Üniversitesi, Ankara. 202.

Amini, M., Younesi ,H., Bahramifar, N., Lorestani, A.A.Z., Ghorbani, F., Daneshi, A., Sharifzadeh, M., 2008. Application of Response Surface Methodology for Optimization of Lead Biosorption in An Aqueous Solution by Aspergillus niger, Journal of Hazardous Materials, 154, 694-702.

Arifiyanto, A., Apriyanti, F. D., Purwaningsih, P., Kalqutny, S. H., 2016. Lead (Pb) bioaccumulation; genera Bacillus isolate $\mathrm{S} 1$ and SS19 as a case study. Proceeding of Internatıonal Bıology Conference Biodiversity and Biotechnology for Human Welfare DOI: 10.1063/1.4985394

Azza, A. A., Wesam, A. H., Hedayat, M. S., Ghada, A. F., 2009. Biosorption of some heavy metal ions using bacterial species isolated from agriculture waste water drains in Egypt. Journal of Applied Sciences Research, 5, 372-383.

Başkaya, H. ve Kocaer, O. 2003. Metallerle kirlenmiş toprakların temizlenmesinde uygulanan teknolojiler, Uludağ Üniversitesi Mühendislik-Mimarlık Fakültesi Dergisi, 8, 121130.

Blindauer, C. A., Harrison, M. D., Robinson, A. K., Parkinson, J. A., Bowness, P. W., Sadler, P. J. and Robinson, N. J., 2002. Multiple bacteria encode metallothioneins and SmtA-like zinc fingers. Molecular microbiology, 45, 14211432.

Blume, H. P., and Hellriegel, T., 1981. Blei-und Cadmium-Status Berliner Böden. Zeitschrift für Pflanzenernährung und Bodenkunde, 144, 181196.

Garnham, G. W., Codd, G. A. and Gadd, G. M., 1992. Kinetics of uptake and intracellular location of cobalt, manganese and zinc in the 
estuarine green alga Chlorella salina. Applied Microbiology and Biotechnology, 37, 270-276.

Heil, D. M., Samani, Z., Hanson, A. T. and Rudd, soil by EDTA. I. Batch and column studies. Water, Air, and Soil Pollution, 113, 77-95.

Kloke, A., Sauerbeck, D. R. and Vetter, H., 1984. The contamination of plants and soils with heavy metals and the transport of metals in terrestrial food chains. In Changing metal cycles and human health, 113-141.

Leung, W.C., Wong, M.F., Chua, H., Co, W., Yu, P.H.F., Leung, C.K., 2000. Removal and recovery of heavy metals by bacteria isolated from activated sludge treating industrial effluents and municipal wastewater. Water Science and Technology, 41, 233-240.

Liu, T., Nakashima, S., Hirose, K., Uemura, Y., Shibasaka, M., Katsuhara, M. and Kasamo, K., 2003. A metallothionein and CPx-ATPase handle heavy-metal tolerance in the filamentous Cyanobacterium Oscillatoria brevis. FEBS letters, 542, 159-163.

Mejáre, M. and Bülow, L., 2001. Metal-binding proteins and peptides in bioremediation and phytoremediation of heavy metals. TRENDS in Biotechnology, 19, 67-73.

Moutsatsou, A., Gregou, M., Matsas, D. and Protonotarios, V., 2006. Washing as a remediation technology applicable in soils heavily polluted by mining-metallurgical activities. Chemosphere, 63, 1632-1640.

Moore, B.A., Duncan, J.R., Burgess, J.E., 2007, ",Fungal Bioaccumulation of Copper, Nickel, Gold and Plantinum, Minerals Engineering, 21, 1-6.

Murthy, S., Geetha, B., Sarangi, SK., 2011. Effect of lead on metallothionein concentration in leadresistant bacteria Bacillus cereus Isolated from Industrial Effluent. African Journal of Biotechnology, 10, 15966-1597.

Murthy, S., Geetha, B., and Sarangi, SK., 2012. Biosorption of lead by Bacillus cereus isolated from Industrial effluents. British Biotechnology Journal, 2, 73-84, 2012.

Naik, M. M., Shamim, K. and Dubey, S. K., 2012. Biological characterization of lead-resistant bacteria to explore role of bacterial metallothionein in lead resistance. Current Science, 426-429.

Naik, M. M. and Dubey, S. K., 2013. Lead resistant bacteria: lead resistance mechanisms, their applications in lead bioremediation and biomonitoring. Ecotoxicology and Environmental Safety, 98, 1-7.

Nies, D. H., 1999. Microbial heavy-metal resistance. Applied microbiology and biotechnology, 51, 730-750.

Özbek H., Kaya Z., Gök M. ve Kaptan, H., 1993. Toprak Bilimi. Çukurova Üniversitesi, Ziraat Fakültesi Genel Yayın, 585-592.

Roane, T. M., 1999. Lead resistance in two bacterial isolates from heavy metalcontaminated soils. Microbial Ecology, 37, 218224.

Roychowdhury,R., Roy, M., Zaman, S., Mitra, A., 2019, Bıoremediation potential of microbes towards heavy metal contamınation, IJRAR, 6, 1088-1092.

Sharma, S., Sundaram, C. S., Luthra, P. M., Singh, Y., Sirdeshmukh, R. and Gade, W. N., 2006. Role of proteins in resistance mechanism of Pseudomonas fluorescens against heavy metal induced stress with proteomics approach. Journal of Biotechnology, 126, 374-382.

Srinath, T., Verma, T., Ramteke, P. W. and Garg, S. K., 2002. Chromium (VI) biosorption and 
bioaccumulation by chromate resistant bacteria. Chemosphere, 48, 427-435.

Srivastava, N. K. and Majumder, C. B., 2008. Novel biofiltration methods for the treatment of heavy metals from industrial wastewater. Journal of Hazardous Materials, 151, 1-8.

Turner, J. S., Glands, P. D., Samson, A. C. and Robinson, N. J., 1996. Zn 2+-sensing by the cyanobacterial metallothionein repressor SmtB: different motifs mediate metal-induced protein-DNA dissociation. Nucleic Acids Research, 24, 3714-3721. 